\title{
Correction to: Is the new ASNM intraoperative neuromonitoring supervision "guideline" a trustworthy guideline? A commentary
}

\author{
Stanley A. Skinner ${ }^{1}$ - Elif Ilgaz Aydinlar ${ }^{2}$. Lawrence F. Borges ${ }^{3} \cdot$ Bob S. Carter $^{4} \cdot$ Bradford L. Currier $^{5}$. \\ Vedran Deletis ${ }^{6} \cdot$ Charles Dong $^{7} \cdot$ John Paul Dormans $^{8} \cdot$ Gea Drost $^{9} \cdot$ Isabel Fernandez-Conejero $^{10}$. \\ E. Matthew Hoffman ${ }^{11}$. Robert N. Holdefer ${ }^{12}$. Paulo Andre Teixeira Kimaid ${ }^{13}$. Antoun Koht ${ }^{14}$. Karl F. Kothbauer ${ }^{15}$. \\ David B. MacDonald ${ }^{16}$. John J. McAuliffe $\mathrm{III}^{17}$. David E. Morledge ${ }^{18}$. Susan H. Morris ${ }^{19}$. Jonathan Norton ${ }^{20}$. \\ Klaus Novak ${ }^{21}$. Kyung Seok Park ${ }^{22}$. Joseph H. Perra ${ }^{23}$. Julian Prell ${ }^{24}$. David M. Rippe ${ }^{25}$. Francesco Sala ${ }^{26}$. \\ Daniel M. Schwartz ${ }^{27} \cdot$ Martín J. Segura $^{28} \cdot$ Kathleen Seidel $^{29} \cdot$ Christoph Seubert $^{30} \cdot$ Mirela V. Simon $^{31}$. \\ Francisco Soto ${ }^{32}$. Jeffrey A. Strommen ${ }^{33,34}$. Andrea Szelenyi ${ }^{35}$. Armando Tello ${ }^{36}$. Sedat Ulkatan ${ }^{37}$. Javier Urriza ${ }^{38}$. \\ Marshall Wilkinson ${ }^{39}$
}

Published online: 16 February 2019

(C) The Author(s) 2019

\section{Correction to: Journal of Clinical Monitoring and Computing https://doi.org/10.1007/s10877-018-00242-3}

The article Is the new ASNM intraoperative neuromonitoring supervision "guideline" a trustworthy guideline? A commentary, written by Stanley A. Skinner, Elif Ilgaz Aydinlar, Lawrence F. Borges, Bob S. Carter, Bradford L. Currier, Vedran Deletis, Charles Dong, John Paul Dormans, Gea Drost, Isabel Fernandez-Conejero, E. Matthew Hoffman, Robert N. Holdefer, Paulo Andre Teixeira Kimaid, Antoun Koht, Karl F. Kothbauer, David B. MacDonald, John J. McAuliffe III, David E. Morledge, Susan H. Morris, Jonathan Norton, Klaus Novak, Kyung Seok Park, Joseph H. Perra, Julian Prell, David M. Rippe, Francesco Sala, Daniel M. Schwartz, Martín J. Segura, Kathleen Seidel, Christoph Seubert, Mirela V. Simon, Francisco Soto, Jeffrey A.
Strommen, Andrea Szelenyi, Armando Tello, Sedat Ulkatan, Javier Urriza and Marshall Wilkinson, was originally published electronically on the publisher's internet portal (currently SpringerLink) on 05 January 2019 without open access. With the author(s)' decision to opt for Open Choice the copyright of the article changed on 30 January 2019 to (c) The Author(s) 2019 and the article is forthwith distributed under the terms of the Creative Commons Attribution 4.0 International License (http://creativecommons.org/licenses/ by/4.0/), which permits use, duplication, adaptation, distribution and reproduction in any medium or format, as long as you give appropriate credit to the original author(s) and the source, provide a link to the Creative Commons license and indicate if changes were made. The original article has been corrected.

The original article can be found online at https://doi.org/10.1007/ s10877-018-00242-3.

Stanley A. Skinner

Stanley.Skinner@allina.com

Extended author information available on the last page of the article 


\section{Affiliations}

Stanley A. Skinner ${ }^{1}$ - Elif Ilgaz Aydinlar ${ }^{2}$ - Lawrence F. Borges ${ }^{3}$ - Bob S. Carter ${ }^{4}$ - Bradford L. Currier ${ }^{5}$. Vedran Deletis ${ }^{6} \cdot$ Charles Dong $^{7} \cdot$ John Paul Dormans ${ }^{8} \cdot$ Gea Drost $^{9} \cdot$ Isabel Fernandez-Conejero $^{10}$. E. Matthew Hoffman ${ }^{11}$ • Robert N. Holdefer ${ }^{12}$. Paulo Andre Teixeira Kimaid ${ }^{13}$. Antoun Koht ${ }^{14} \cdot$ Karl F. Kothbauer $^{15}$. David B. MacDonald ${ }^{16}$. John J. McAuliffe $\mathrm{III}^{17}$. David E. Morledge ${ }^{18}$. Susan H. Morris ${ }^{19}$. Jonathan Norton ${ }^{20}$. Klaus Novak ${ }^{21} \cdot$ Kyung Seok Park ${ }^{22}$. Joseph H. Perra ${ }^{23}$. Julian Prell ${ }^{24}$ • David M. Rippe ${ }^{25}$. Francesco Sala ${ }^{26}$. Daniel M. Schwartz ${ }^{27} \cdot$ Martín J. Segura $^{28} \cdot$ Kathleen Seidel $^{29} \cdot$ Christoph Seubert $^{30} \cdot$ Mirela V. Simon $^{31}$. Francisco Soto ${ }^{32}$. Jeffrey A. Strommen ${ }^{33,34}$. Andrea Szelenyi ${ }^{35}$. Armando Tello ${ }^{36}$. Sedat Ulkatan ${ }^{37}$. Javier Urriza ${ }^{38}$. Marshall Wilkinson ${ }^{39}$

1 Intraoperative Neurophysiology Department, Abbott Northwestern Hospital, Minneapolis, MN, USA

2 Acibadem University School of Medicine, Department of Neurology, Istanbul, Turkey

3 Massachusetts General Hospital, Harvard Medical School, Boston, MA, USA

4 Department of Neurosurgery, Harvard Medical School, Massachusetts General Hospital, Boston, MA, USA

5 Department of Orthopedics, Mayo Clinic, Rochester, MN, USA

6 Albert Einstein College of Medicine, New York, NY, USA

7 Division of Neurosurgery, Department of Surgery, University of British Columbia, Vancouver, BC, Canada

8 Pediatric Orthopedic Surgery, Riley Hospital for Children, Indiana University School of Medicine, Indianapolis, IN, USA

9 Department of Neurosurgery/Neurology, University Medical Center Groningen, Groningen, The Netherlands

10 Department of Intraoperative Neurophysiology, Hospital Universitari de Bellvitge, L'Hospitalet de Llobregat, Barcelona, Spain

11 Department of Neurology, Mayo Clinic, Rochester, MN, USA

12 Department of Rehabilitation Medicine, University of Washington, Harborview Medical Center, Seattle, WA, USA

13 Department of Neurosurgery, Federal University of São Paulo, São Paulo, Brazil

14 Feinberg School of Medicine, Northwestern University, Chicago, IL, USA

15 Division of Neurosurgery, Luzerner Kantonsspital, University of Basel, 6000 Lucerne, Switzerland

16 Department of Neurosciences, Section of Neurophysiology, King Faisal Specialist Hospital \& Research Center, MBC 76, PO Box 3354, Riyadh 11211, Saudi Arabia

17 Department of Anesthesia, Cincinnati Children's Hospital, The University of Cincinnati, Cincinnati, OH, USA

18 Neurostatus, Eagle, ID, USA

19 IWK Children's Health Program \& Division of Neurosurgery, Dalhousie University, Halifax, NS, Canada

20 Department of Surgery, University of Saskatchewan, Saskatoon, SK, Canada
21 Department of Neurosurgery, Medical University of Vienna, Vienna, Austria

22 Department of Neurology, Seoul National University Bundang Hospital, Seoul National University College of Medicine, Seongnam, Republic of Korea

23 Twin Cities Spine Center, Minneapolis, MN, USA

24 Department of Neurosurgery, Halle (Saale), Germany

25 Neurophysiology Department, Abbott Northwestern Hospital, Minneapolis, MN, USA

26 Section of Neurosurgery, Department of Neurosciences, Biomedicine and Movement Sciences, University Hospital, Verona, Italy

27 Surgical Monitoring Associates, Springfield, PA, USA

28 Clinical Neurophysiology Unit, Hospital Garrahan, University of Buenos Aires Medical School, Buenos Aires, Argentina

29 Intraoperative Neurophysiology and Neuro-oncological Surgery, Department of Neurosurgery, Inselspital Bern University Hospital, Bern, Switzerland

30 Division of Neuroanesthesia, College of Medicine, University of Florida, Gainesville, FL, USA

31 Intraoperative Neurophysiology Unit, Department of Neurology, Harvard Medical School, Massachusetts General Hospital, Boston, MA, USA

32 Neurology Department, Clínica Las Condes, Santiago, Chile

33 Department of Physical Medicine and Rehabilitation, Mayo Clinic, Rochester, MN, USA

34 Neurophysiology Department, Abbott Northwestern Hospital, Minneapolis, MN, USA

35 Clinical and Intraoperative Neurophysiology, Department of Neurosurgery, Hospital of University of Munich, Ludwig Maximilians Universitaet Muenchen (LMU), Munich, Germany

36 Clinical Neurophysiology Department, Hospital Español, Mexico City, Mexico

37 Intraoperative Monitoring Service, Mount Sinai West Hospital, New York, NY, USA

38 Intraoperative Neurophysiology Unit, Clinical Neurophysiology Department, Complejo Hospitalario de Navarra - B, Pamplona-Iruña, Navarra, Spain

39 Section of Neurosurgery, University of Manitoba, Winnipeg, MB, Canada 